\title{
Sensitivity Analysis and Effect of Simulation parameters of CPFD Simulation in Fluidized Beds
}

\author{
Janitha C. Bandara $^{1} \quad$ Henrik K. Nielsen $^{2} \quad$ Britt M.E. Moldestad $^{1} \quad$ Marianne S. Eikeland $^{1}$ \\ ${ }^{1}$ Department of Process, Energy and Environmental Technology, University College of Southeast Norway, Norway, \\ \{Janitha.bandara, britt.moldestad, Marianne.Eikeland\}@usn.no \\ ${ }^{2}$ Department of Engineering Sciences, University of Agder, Norway, \\ \{henrik.kofoed.nielsen\} Quia.no
}

\begin{abstract}
Fluidized bed technology is broadly applied in industry due to its distinct advantages. CFD simulation of fluidized beds is still challenging compared to singlephase systems and needs extensive validation. Multiphase particle-in-cell is a recently developed lagrangian modeling technique and this work is devoted to analyze the sensitivity of grid size, time step, and model parameters, which are the essences of accurate results. Barracuda VR 17.1.0 commercial CFD package was used in this study.

$500 \mu \mathrm{m}$ sand particles and air was used as the bed material and fluidization gas respectively. Five different grids, having 27378, 22176, 16819, 9000 and 6656 computational cells were analysed, where five different time steps of $0.05,0.01,0.005,0.001$ and 0.0005 were used for each grid. One velocity step was maintained for 8 seconds. The bed pressure drop at packed bed operation was high for simulations with reduced time steps while equal pressure drops were observed during fluidization for all time steps. Time steps of $0.0005 \mathrm{~s}$ and $0.001 \mathrm{~s}$ and $0.005 \mathrm{~s}$ produced equal result of $0.15 \mathrm{~m} / \mathrm{s}$ for minimum fluidization velocity, irrespective of the grid size. The results from time steps of 0.05 and 0.01 are converged to the results from time steps of 0.005 and 0.001 by increasing simulation time per one velocity step.
\end{abstract}

Keywords: $\quad$ Fluidized bed, Minimum fluidization velocity, CFD simulations, Multiphase particle-in-cell method, Grid size, Time step

\section{Introduction}

Gas-solid fluidized bed technology is widely utilized in energy generation, chemical, petrochemical, pharmaceutical, environmental, electronic and metallurgical processing industries due its distinct advantages of high heat and mass transfer, controlled material handling, large thermal inertia of solids and isothermal operating conditions (de Souza Braun et al. 2010)(Vejahati et al. 2009)(Esmaili and Mahinpey 2011). Computational fluid dynamic (CFD) modeling has been identified as an excellent tool to produce missing information during the scaling up of lab/pilot scale fluidized beds to industrial scale. Further, it is a fast and cost effective method for system optimization.

CFD solves the conservation equations for mass, momentum, energy and species where this technique has been critically validated for the accurate performance in gas or liquid single phase flows. However, there are certain challenges related to interface coupling, solid phase modeling and scale differences in gas-solid multiphase flow systems. Eulerian-eulerian and eulerian-lagrangian are the two basic approaches for CFD modeling of multiphase flows.

Multiphase Particle-In-Cell (MP PIC) modeling is a development of eulerian-lagrangian modeling and aims to reduce the computational cost in discrete modeling of particle phase. Instead of tracking individual particles, it considers the parcels containing a certain number of particles with similar properties. The parcels are modeled in the discrete phase while the particle phase interactions are modeled in Eulerian frame. Therefore particle properties are calculated in both Eulerian and Lagrangian frames, which are correlated via interpolation functions. The successive developments of the MP-PIC method is illustrated in the works of Snider, O'Rourke and Andrew (Andrews and O'Rourke 1996)(D M Snider 2001)(D M Snider, O'Rourke, and Andrews 1998)(Dale M Snider 2007)(O'Rourke and Snider 2012).

Validated CFD models can be used to analyze the bubbling fluidized beds in terms of minimum fluidization velocity, bubble rise velocity, bubble diameter and particle mixing and segregation. The conservation equations of mass, species, momentum and energy are in partial differential form. The particular simulation geometry is divided into small cells, which is referred as the computational grid. The conservation equations are then discretized in space and time to get a set of algebraic equations. Finite difference, finite element and finite volume are the main techniques where the finite volume method is mostly used in mass, momentum and energy related 3D systems.

Errors and uncertainties are integrated from the modeling stage to the final computer simulations. Use of empirical equations and model simplification lead to deviations during the model development. The errors 
imposed due to the selection of mesh size, time step and discretization method are referred as numerical errors. Truncation and limiting functions at the discontinuities also cause deviations in the result. Iterative algorithms used in simulations provide certain errors while the round off errors are integrated depending upon computer resource (i.e. 32 bit or 64 bit). Finally, improper coding can also lead to certain errors where these are absorbed as discretization errors. Therefore, it is required to identify the possibilities to reduce the errors in the simulations with minimal computational cost.

As the model equations are concerned, it is possible to check the best functioning empirical models. This includes selecting the best drag model in gas-solid multiphase flow systems. Checking different values for the model constants/coefficients in a meaningful way is another approach. Different schemes such as first order upwind, second order upwind and central differencing etc. can be optimized in terms of computer cost and accuracy required. However, many of the mentioned parameters are optimized for general setting in many of the commercial CFD packages. Hence, the most primary parameters to study in first hand are the grid size and the simulation time step. These two parameters are correlated to form Courant-Friedrichs-Lewy (CFL) conditions, which gives the primary indication of the convergence of the simulation. Apart from the convergence, improper implementation of mesh lead to errors and missing information of the systems.

Mesh sensitivity analysis has used to develop a grid independent model. Many of the related works for the mesh sensitivity were carried out for EE simulations and fixed time steps has been adopted based on convergence criteria. In contrast, as solid phase is modeled as discrete particles in EL modeling, the solid phase interactions are directly calculated. Therefore, the effect of the mesh size is comparatively less. Many authors have used the bed pressure drop and the solid volume fraction as the parameters to check the mesh sensitivity. Even though the MP PIC modeling preserves the discrete nature of the particles, it deviates from the original Lagrangian modeling as selected particle properties are calculated in the Eulerian frame.

Barracuda VR is a tailor-made CFD code for multiphase flow systems, which uses MP PIC modeling. Many of the previous studies on sensitivity analysis have been carried out in steady boundary conditions. Instead, this work is focused on studying both changing boundary conditions of inlet flow velocity during the transition of packed bed to fluidized bed and steady boundary conditions in bubbling fluidizing regime. The minimum fluidization velocity, bed pressure drop and bubble characteristics were compared. Barracuda VR 17.1.0 version was used to compare the minimum fluidization velocity, bed pressure drop and bubble characteristics between different configurations of time step, grid size along with different models and model parameters.

\section{MP PIC Model Description}

The gas phase mass and momentum conservation are modeled with continuity and time averaged NaiverStokes equations:

$$
\begin{gathered}
\frac{\partial\left(\alpha_{g} \rho_{g}\right)}{\partial t}+\nabla \cdot\left(\alpha_{g} \rho_{g} u_{g}\right)=0 \\
\frac{\partial\left(\alpha_{g} \rho_{g} u_{g}\right)}{\partial t}+\nabla \cdot\left(\alpha_{g} \rho_{g} u_{g} u_{g}\right)=-\nabla P+F+ \\
\nabla \cdot\left(\alpha_{g} \tau_{g}\right)+\alpha_{g} \rho_{g} g
\end{gathered}
$$

Where $\alpha_{g}, \rho_{g}$, and $u_{g}$ are gas phase volume fraction, density and velocity respectively. $\mathrm{F}$ is total momentum exchange with particle phase per volume, $\mathrm{g}$ is gravitational acceleration, $\mathrm{P}$ is pressure and $\tau_{g}$ is the gas phase stress tensor, which is given by:

$$
\tau_{g}=\mu_{g}\left[\left(\nabla u_{g}+\Delta u_{g}^{T}\right)-\frac{2}{3} \nabla \cdot u_{g} I\right]
$$

$\mu_{g}$ refers to the shear viscosity that is the sum of the laminar and turbulent components. The large eddy simulation is used for the large-scale turbulence modeling while the subgrid scale turbulence is captured with Smagorinsky model:

$$
\mu_{g, t}=C_{s} \rho_{g} \Delta^{2}\left|\nabla u_{g}+\Delta u_{g}^{T}\right|
$$

Where $\Delta$ is the subgrid length scale and calculated by equation 05 . The default value for the model constant $C_{S}$ is 0.01 .

$$
\Delta=(\delta x \delta y \delta z)^{1 / 3}
$$

The interface momentum transfer is calculated through the viscous drag force:

$$
F=\iint f\left\{m_{p}\left[D_{p}\left(u_{g}-u_{p}\right)-\frac{\nabla P}{\rho_{p}}\right]\right\} d m_{p} d u_{p}
$$

Subscript $P$ refers to the particle phase properties where $\mathrm{m}$ and $\mathrm{u}$ symbolizes the mass and velocity. $D_{p}$ is the drag function. The particle phase dynamics are derived using particle distribution function (PDF) calculated from the Liouville equation given as:

$$
\frac{\partial f}{\partial t}+\nabla\left(f u_{p}\right)+\nabla u_{p}\left(f A_{p}\right)=0
$$

Where $A_{p}$, is the particle acceleration and is expressed by:

$$
A_{p}=\frac{\partial\left(u_{p}\right)}{\partial t}=D_{p}\left(u_{g}-u_{p}\right)-\frac{\nabla P}{\rho_{p}}-\frac{\nabla \tau_{p}}{\rho_{p} \alpha_{p}}+g
$$


$\alpha_{p}$ is particle volume fraction and $\tau_{p}$ is particle stress function that is used in formulating interphase interactions of particles.

$$
\begin{gathered}
\alpha_{p}=\iint f \frac{m_{p}}{\rho_{p}} d m_{p} d u_{p} \\
\tau_{p}=\frac{10 P_{s} \alpha_{p}^{\beta}}{\max \left[\left(\alpha_{c p^{-}}-\alpha_{p}\right), \varepsilon\left(1-\alpha_{p}\right)\right]}
\end{gathered}
$$

$P_{s}$ is a constant with the units of pressure, $\alpha_{c p}$ is the particle volume fraction at close packing, $\beta$ is a constant between 2 and 5 where $\varepsilon$ is a very small number on the order of $10^{-7}$.

\section{Methods and Computational Model}

The minimum fluidization velocity (MFV) was used as the primary measurement for the mesh and time step sensitivity analysis. The simulations were started at the packed bed conditions and the gas velocity was gradually increased from zero to $0.4 \mathrm{~m} / \mathrm{s}$ with 0.025 increments. Simulations were carried out for 8 seconds at each velocity step. Average pressure drop gradient across the column was plotted against the gas superficial velocity and the minimum fluidization velocity is read $\left(U_{m f}\right)$ as illustrated in Figure 1. Five different grid sizes and five different time steps for each grid were used to compare the MFVs. The simulation time for each velocity step was gradually increased in the following simulations gradually up to 20 seconds in selected grids and the results were compared. As the drag model is a function of particle volume fraction, the MFV was analyzed at varied close pack volume fractions.

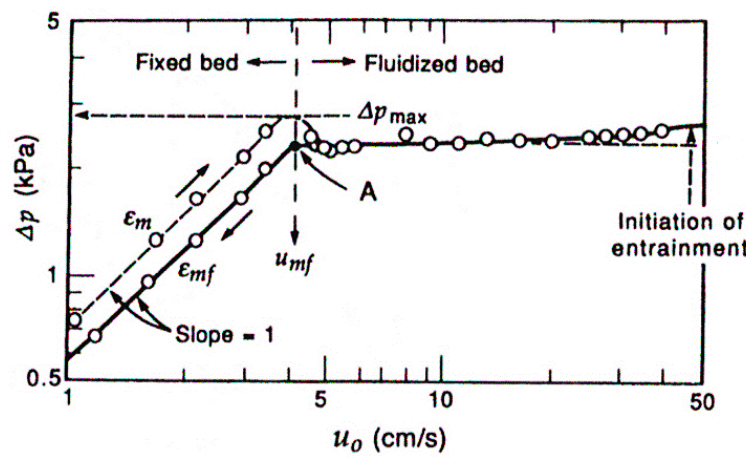

Figure 1. Calculation of minimum fluidization velocity

\subsection{Computational model}

The dimensions of the geometry were adopted from the experimental rig at the University of Southeast Norway. As shown in Figure 2, a cylindrical column with $84 \mathrm{~mm}$ in diameter and $1000 \mathrm{~mm}$ in height with pressure monitoring points in $100 \mathrm{~mm}$ intervals along the height was created. The gas inlet was set up as a flow boundary while the top gas exit as a pressure boundary at atmospheric pressure with no particle exit. Fluidizing gas was air at $300 \mathrm{~K}$ with varying superficial velocity.
Further, the velocity inlet was formulated as it homogeneously injects air in axial direction throughout the whole bottom cross section. Each velocity was maintained for 8 seconds. Spherical sand particles with $2200 \mathrm{Kg} / \mathrm{m}^{3}$ in density and 500 micron in diameter was the bed material used. The initial particle bed height was set up to $350 \mathrm{~mm}$.

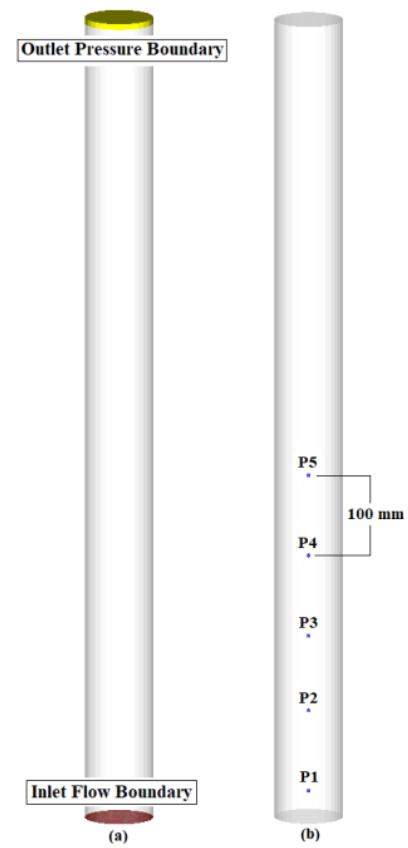

Figure 2. (a) Boundary conditions, (b) Pressure points Five different meshes with 6656, 9000, 16819, 22176 and 27378 cells were tested and cross sectional views are illustrated in Figure 3.

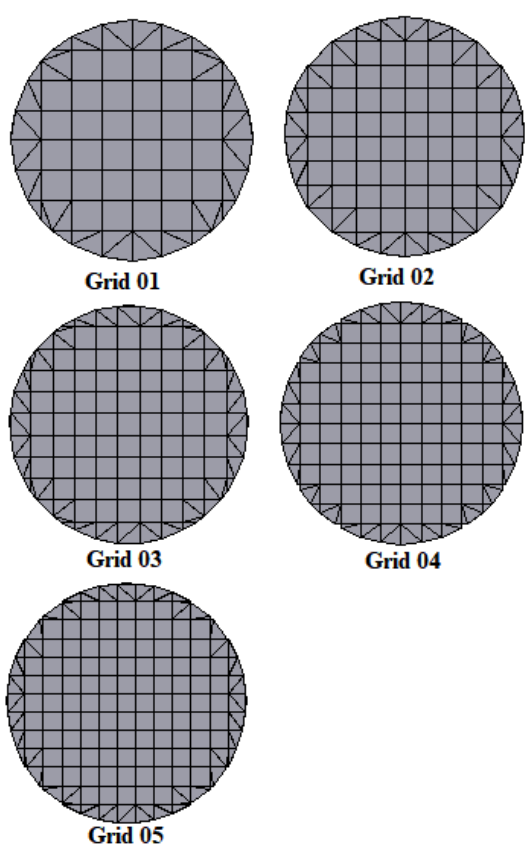

Figure 3. Cross sectional views of different grids

The grid dimensions in $\mathrm{x}, \mathrm{y}$ and $\mathrm{z}$ direction for each mesh are given in Table 1. The normalized grid size in all $\mathrm{x}, \mathrm{y}$ and $\mathrm{z}$ directions were kept below the warning 
line in the grid check plot. Grid refinements at the wall was not performed as it was assumed that there was no boundary layer formation with the dense phase particle system. Default grid generator settings were used, which removes the cells having less fraction of volume than 0.04 and greater aspect ratio than 15:1. Four time steps of $0.05,0.01,0.005$ and 0.001 seconds were checked for each grid.

Table 1. Cell dimensions

\begin{tabular}{lllll}
\hline $\begin{array}{l}\text { No of } \\
\text { cells }\end{array}$ & $\begin{array}{l}\Delta X \\
(\mathrm{~mm})\end{array}$ & $\begin{array}{l}\Delta Y \\
(\mathrm{~mm})\end{array}$ & $\begin{array}{l}\Delta Z \\
(\mathrm{~mm})\end{array}$ & $\begin{array}{l}\text { Grid } \\
\text { No }\end{array}$ \\
\hline 6656 & 10.5 & 10.5 & 9.6 & 01 \\
\hline 9000 & 8.40 & 8.40 & 11.1 & 02 \\
\hline 16819 & 7.60 & 7.60 & 7.20 & 03 \\
\hline 22176 & 7.00 & 7.00 & 6.5 & 04 \\
\hline 27378 & 6.46 & 6.46 & 6.17 & 05 \\
\hline
\end{tabular}

Adopting to the previous experience of the author (Bandara, Thapa, Moldestad, \& Eikeland, 2016), WenYu-Ergun correlation was used for the initial simulations. It is a combined formulation of Wen-Yu model and Ergun model, which is selected upon the gas volume fraction. When the gas volume fraction is greater than 0.8 , Wen-Yu correlation is applied which is given by,

$$
K_{\frac{s g}{W e n_{Y u}}}=\frac{3}{4} \frac{C_{d} \rho_{g} \varepsilon_{g}\left(1-\varepsilon_{g}\right)\left(u_{s}-u_{g}\right)}{d_{p}} \varepsilon_{g}^{-2.65}
$$

Where $C_{d}$ is given by,

$$
C_{d}=\left\{\begin{array}{rr}
\frac{24}{\varepsilon_{g} R e_{s}}\left[1+0.15\left(\varepsilon_{g} R e_{s}\right)^{0.687}\right], & R e_{s} \leq 1000 \\
0.44, & R e_{s}>1000
\end{array}\right.
$$

When the gas volume fraction is less than 0.8, Ergun correlation is used which is given by,

$$
K_{\frac{s g}{E r g u n}}=150 \frac{\mu_{g}\left(1-\varepsilon_{g}\right)^{2}}{\varphi^{2} d_{p}^{2} \varepsilon_{g}}+1.75 \frac{\rho_{g}\left(u_{g}-u_{s}\right)\left(1-\varepsilon_{g}\right)}{\varphi d_{p}}
$$

Where, subscripts $g, p$ and s refer to gas phase, particle and solid phase respectively. $\mathrm{K}_{\mathrm{sg}}$ is the interface momentum transfer coefficient, $U$ is the velocity, $\rho$ is the density, $\varepsilon$ is the volume fractions, $\varphi$ is the sphericity, $\mu$ is the viscosity, $R e$ is the Reynold's number and $d$ is the particle diameter.

The close pack volume fraction, maximum momentum redirection from collisions, normal to wall momentum retention and tangent to wall momentum retention were set to $0.6,40 \%, 0.3$ and 0.99 respectively. Default values for the parameters in the particle stress model were kept unchanged. Large eddy simulation was enabled for the turbulence modeling and "partial-donorcell" was used as the numerical scheme.

\section{Results and Discussion}

Minimum fluidization is a crucial parameter as it represents the minimum gas required to operate the reactor. It is sensitive to particle properties (size, shape, density etc.) and gas properties (density, humidity, viscosity etc.) along with geometry (aspect ratio). Therefore, it is required to know the minimum fluidization velocity at different contexts. A CFD model can be useful in predicting MFV at various process conditions. This work demonstrates the grid size and time step dependency in calculating the minimum fluidization velocity.

Apart from the 20 simulations mentioned under the methods, time step of 0.0005 was used for grids with high resolutions and one other simulation was carried out at a coarse grid. The plots were generated for each grid at different time steps and each time step for different grids.

According to the force balance at the minimum fluidization condition, the bed pressure drop is proportional to the particle weight and can be expressed as,

$$
\frac{\Delta P}{H}=\left(1-\varepsilon_{m f}\right) g\left(\rho_{s}-\rho_{g}\right)
$$

Where, $\Delta P$ is bed pressure drop, $H$ is bed height and $\varepsilon_{m f}$ is the void fraction at the minimum fluidization.

Equation 14 and many correlations for the MFV need the knowledge of void fraction at minimum fluidization, which is difficult to determine. However, Gidaspow (1994) and Das et al have mentioned about the void fraction at minimum fluidization $\left(\varepsilon_{m f}\right)$, which varies between 0.44 and 0.476 . Implementing the value of 0.45 for the $\varepsilon_{m f}$ in equation 14, the pressure drop per unit height of the bed at minimum fluidization is 11.87 $\mathrm{Pa} / \mathrm{mm}$.

As approximated by Wen and $\mathrm{Yu}$, the minimum fluidization velocity $U_{m f}$ can be expressed as,

$$
U_{m f}=\frac{\mu_{g}}{\rho_{g} d_{p}}[\sqrt{1135.7+0.048 A r}-33.7]
$$

Where $\mu_{g}$ is gas viscosity, $d_{p}$ is particle diameter and $A r$ is the Archimedes number given by,

$$
A r=\frac{d_{p}^{3}\left(\rho_{s}-\rho_{g}\right) \rho_{g} g}{\mu^{2}}
$$

Using Equation 15 and 16, the MFV for the simulated system can be calculated as $0.165 \mathrm{~m} / \mathrm{s}$.

Air velocity $\left(u_{g}\right)$ vs pressure drop $(\Delta P)$ plots for different grids are illustrated in Figure 4 to 8 . Each figure contains plots for different time steps used. Each velocity step was maintained for 8 seconds and the pressure drop was taken as the average value of the $8^{\text {th }}$ second of respective velocity. The averaging was performed to minimize the effect of random pressure fluctuations during fluidization on results. The pressure gradient $(\mathrm{Pa} / \mathrm{mm})$ along the column height was 
calculated based on P1 and P2 data as illustrated in sketch (b) - Figure 2. It was assumed that the P1 and P2 would reach the steady values before the rest of the transient data points (P3, P4 and P5) for pressure.

\subsection{Bed Pressure drop}

Being a fundamental formulation, pressure drop at onset of fluidization calculated from Equation 14, which is $11.87 \mathrm{~Pa} / \mathrm{mm}$, was used as the baseline to compare the results from simulations.

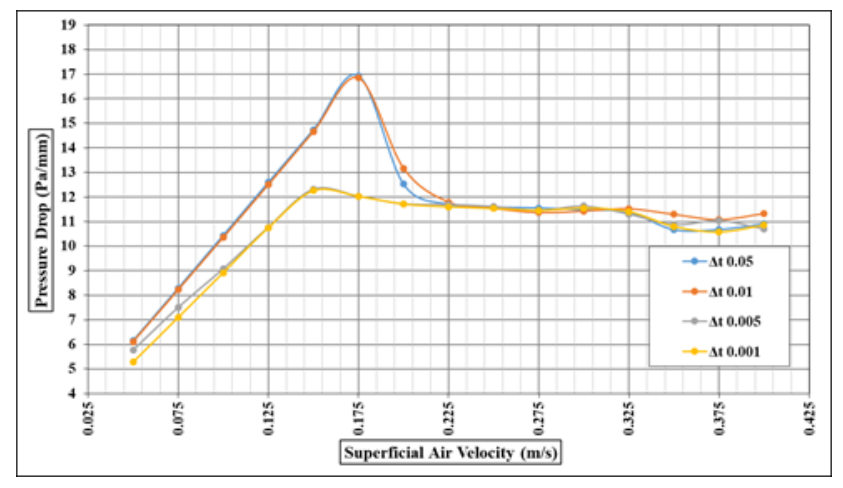

Figure 4. Effect of the time step for MFV at grid 01

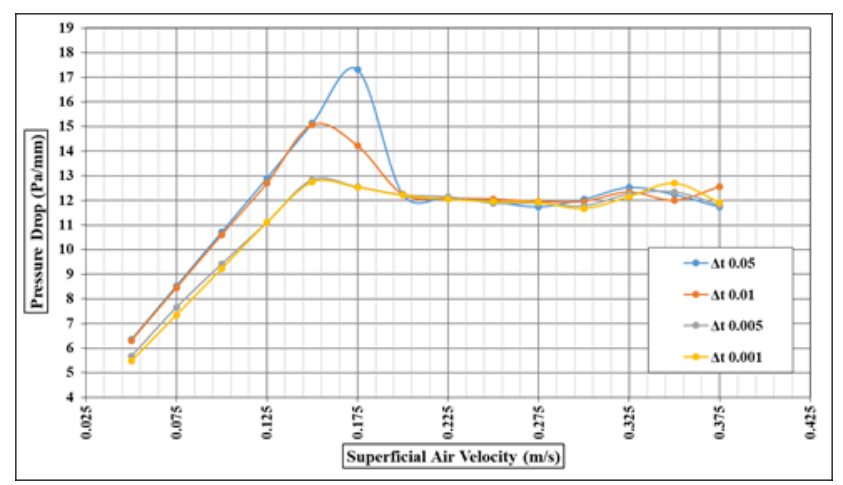

Figure 5. Effect of the time step for MFV at grid 02

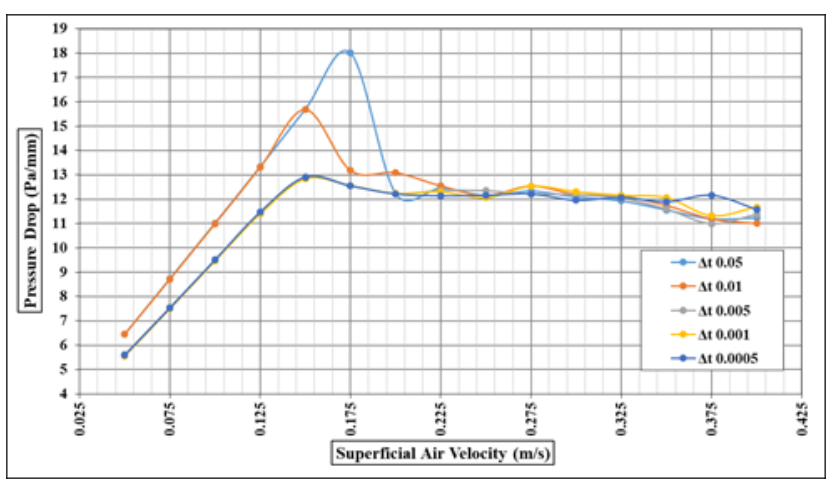

Figure 6. Effect of the time step for MFV at grid 03

\subsubsection{Pressure drop at minimum fluidization}

The results for the pressure drop at minimum fluidization $\left((\Delta P)_{M F}\right)$ using time step 0.05 show the highest variation of $18 \mathrm{~Pa} / \mathrm{mm}$ in grid 05 and 03 . The respective value changes between 17 and $18 \mathrm{~Pa} / \mathrm{mm}$ at different grids without any distinguishable pattern. At the coarsest grid, grid 01 , both time steps of 0.05 and
0.01 give the same of $17 \mathrm{~Pa} / \mathrm{mm}$ for $(\Delta P)_{M F}$. However, the $(\Delta P)_{M F}$ using time step 0.01 gradually increases from $15 \mathrm{~Pa} / \mathrm{mm}$ to $16.5 \mathrm{~Pa} / \mathrm{mm}$ as the grid size is reduced from grid 02 to grid 05 . The $(\Delta P)_{M F}$ calculated from time steps of 0.005 and 0.001 are identical for each grid, which gradually increase from $12.5 \mathrm{~Pa} / \mathrm{mm}$ in grid 01 to $13.5 \mathrm{~Pa} / \mathrm{mm}$ in grid 05 .

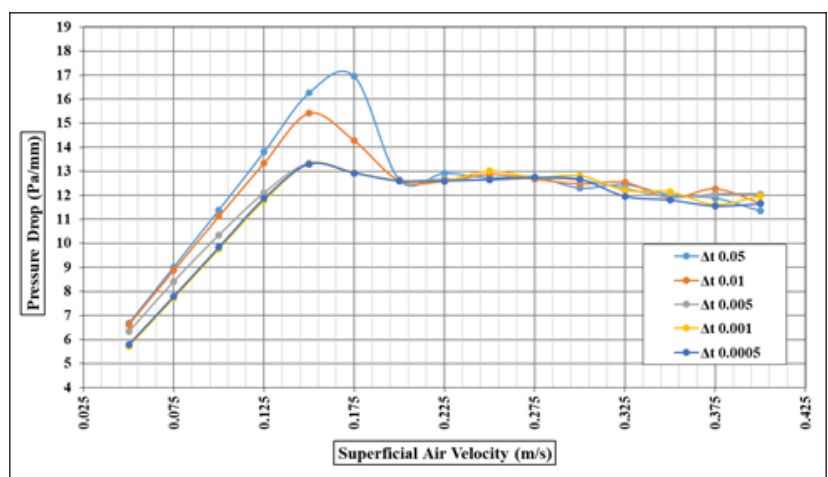

Figure 7. Effect of the time step for MFV at grid 04

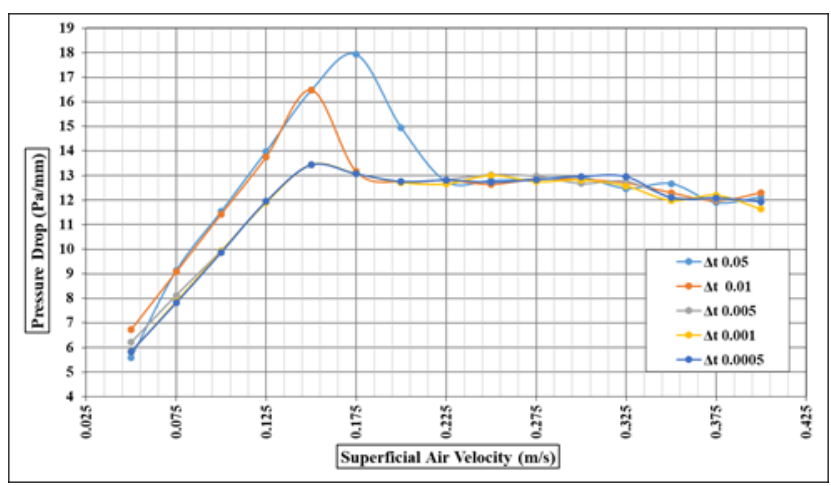

Figure 8. Effect of the time step for MFV at grid 05

\subsubsection{Pressure drop during packed bed}

Simulation results from time steps of 0.05 and 0.01 behaves almost equally at each grid during packed bed operation. The observed $\Delta P \mathrm{~s}$ are considerably higher compared to time steps of 0.005 and 0.001 at each velocity step. The curves from time steps of 0.001 and 0.0005 are identical throughout the full range of air velocities. The $\Delta P$ using time step 0.005 almost follow the time step of 0.001 with slight over prediction in grid 01 and 04 . However, the curve converges to that of the time step of 0.001 before the onset of fluidization.

\subsubsection{Pressure drop at fluidization regime}

The $\Delta P$ during fluidization was similar for all the time steps at each grid. However, respective value increases from $11.5 \mathrm{~Pa} / \mathrm{mm}$ in grid 01 to $13 \mathrm{~Pa} / \mathrm{mm}$ in grid 02 . Almost steady pressure drops can be observed for time steps of $0.0005,0.001$ and 0.005 between $0.2 \mathrm{~m} / \mathrm{s}$ and $0.325 \mathrm{~m} / \mathrm{s}$ air velocities. After $0.325 \mathrm{~m} / \mathrm{s}$ of air velocity, the $\Delta P$ starts to fluctuate for all the simulations. The $\Delta P$ is dropped down nearly by $1 \mathrm{~Pa} / \mathrm{mm}$ after $0.325 \mathrm{~m} / \mathrm{s}$ air velocity except in grid 02 , in which the $\Delta P$ is slightly increased. 


\subsection{Minimum Fluidization Velocity}

The results for minimum fluidization velocity does not show much variations over the grid sizes. The MFV obtained from time step of 0.05 is $0.175 \mathrm{~m} / \mathrm{s}$ for all the grids. Time steps of $0.005,0.001$ and 0.0005 produce the same MFV of $0.15 \mathrm{~m} / \mathrm{s}$ irrespective of the grid size. The time step of 0.01 gives the same MFV velocity of $0.15 \mathrm{~m} / \mathrm{s}$ for grid $02,03,04$ and 05 where in grid 01 , $\mathrm{MFV}$ is increased to $0.175 \mathrm{~m} / \mathrm{s}$.

The observed differences in the $\Delta P$ and MFV might be related to CFL conditions or not reaching steady state conditions at each velocity steps. The CFL equation is given by:

$$
C F L=u \frac{\Delta t}{\Delta x}
$$

Where $\Delta t$ is time step, $\Delta x$ is cell size (one dimensional modeling) and $u$ is the convective flow velocity.

At lower time steps the air flow is not fully developed. This effect is progressively increased along the column height. Due to that, the air velocity is getting lesser along the height, which force the cells near the inlet flow boundary to store more air according to the step wise increment of air velocity. This leads to increased pressures near the inlet boundary and consequently increased pressure drop gradients. Therefore, the pressure gradients along the height are less linear for higher time steps. Hence, selecting the transient data points of P1, P2...P3 (refer sketch (b) in Figure 2) to calculate the pressure drop gradient was critical for previous simulation results. This variation is clearly illustrated in air velocity vs pressure drop plots in Figure 9. The simulation results from grid 03 was used and the pressure gradients were calculated using different transient data points according to the formulations mentioned at the lower right hand corner of each plot. The time steps of 0.005 and 0.001 produce almost same results irrespective of the transient data points used. Even though the $(\Delta P)_{M F}$ is high with time step of 0.05 , all the plots follow a similar trend. In contrast, the curves for time step 0.01 show higher deviations from each other and however, with less $(\Delta P)_{M F}$ compared to time step 0.05 . The collective outcome of these results clearly illustrates that the system has not achieved steady state operation completely with the implemented boundary conditions at lower time steps of 0.05 and 0.01 .

Therefore, further simulations were carried out for time steps of 0.05 and 0.01 with extended simulation time of 14 seconds and 20 seconds for each velocity step. Grid 03 was used and air velocity vs pressure drop plots are illustrated in Figure 10 and Figure 11 along with the results from 8 seconds simulation time.

When the plots in Figure 11 are compared, results from 14 second and 20 second simulation time are converged to same values in terms of both $(\Delta P)_{M F}$ and
MFV. $(\Delta P)_{M F}$ remains at $13.6 \mathrm{~Pa} / \mathrm{mm}$ while the $\mathrm{MFV}$ is further reduced to $0.13 \mathrm{~m} / \mathrm{s}$. This suggests the inability of further improvement of the results merely by increasing the simulation time for time step 0.01 . Therefore, it is necessary to carry out additional simulations with increased simulation time for time steps of 0.005 and 0.001 for other grids to see the provisions for the improvements of the results.

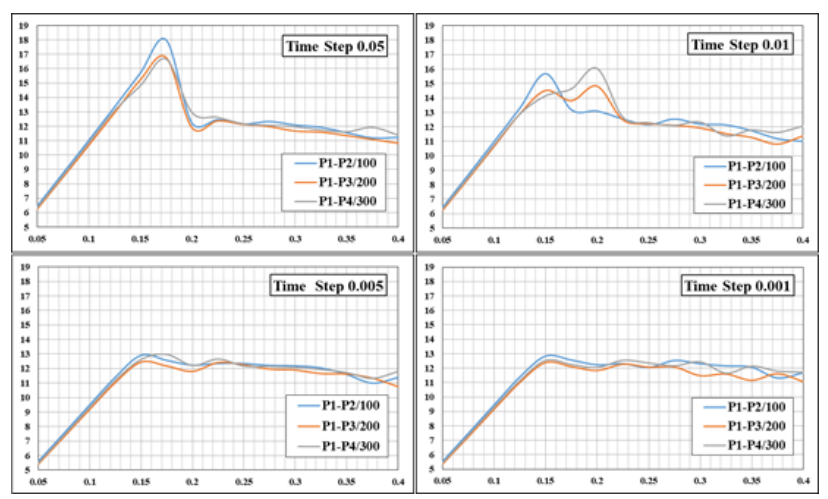

Figure 9. Effect of the time step for MFV at grid 03

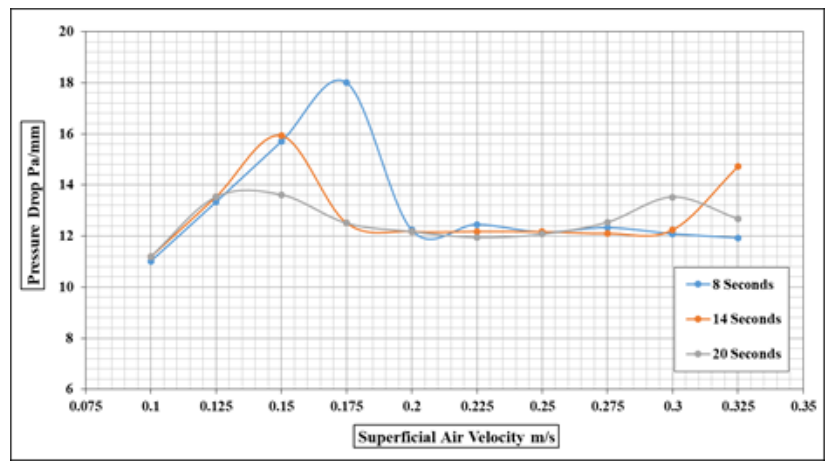

Figure 10. Effect of the simulation time for MFV and pressure drop at time step of 0.05 seconds

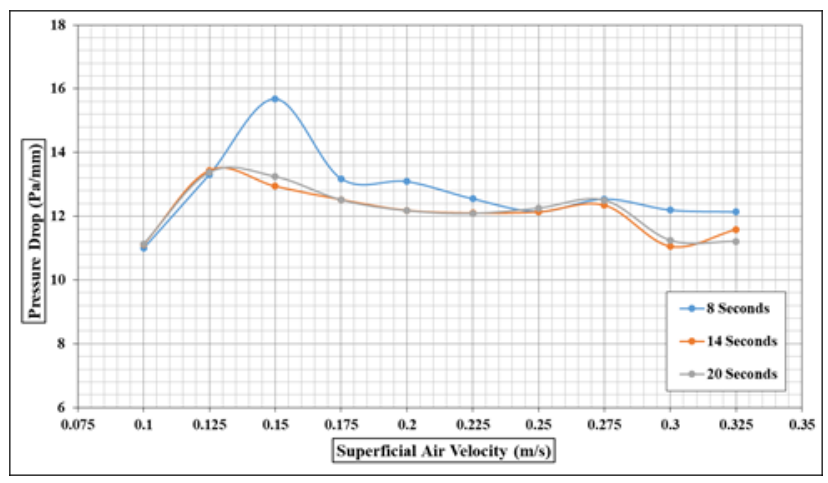

Figure 11. Effect of the simulation time for MFV and pressure drop at time step of 0.01 seconds

\subsection{Effect of the close volume fraction for minimum fluidization velocity}

Most of the drag models are a function of particle volume fraction $\left(\alpha_{p}\right)$, which is changed depending on particle shape and size distribution. The previously illustrated simulation results were based on close volume fraction of 0.6 and successive simulations are 
carried out for $0.55,0.58$ and 0.65 . The results are illustrated in Figure 12.

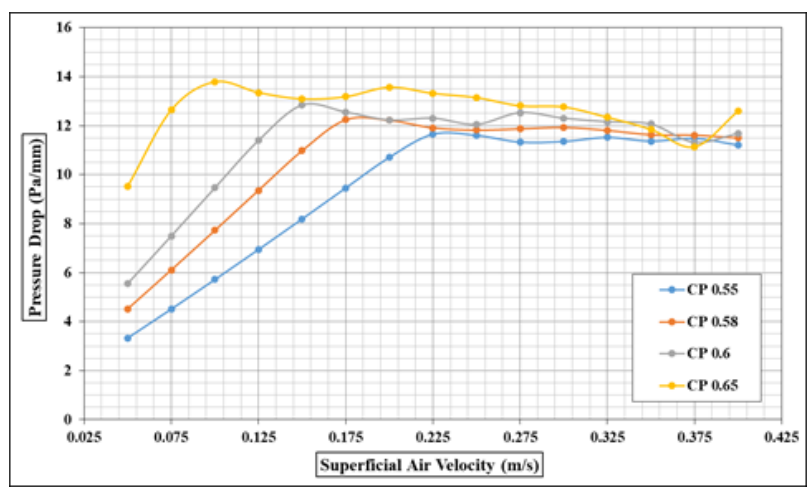

Figure 12. Effect of the close volume fraction for MFV and pressure drop

Grid 03 and time step of 0.001 second were used for the simulations. The pressure drop during packed bed operation is increased with the increased close pack volume fraction. The particles are closely packed that makes it hard for gas to pass through. A slight change in the $(\Delta P)_{M F}$ can also be observed, which is increased proportionally with close volume fraction. There is a significant variation in the MFV, which is reduced down to $0.1 \mathrm{~m} / \mathrm{s}$ at close volume fraction of 0.65 and as high as $0.225 \mathrm{~m} / \mathrm{s}$ at 0.55 . The drag functions are functions of the particle volume fraction which leads to the difference in MFV. The bed pressure drops are converged together as the air velocity is increased. This is because, the densely packed particles are loosened and attain a more or less common particle volume fraction as the system undergoes rigorous fluidization.

\subsection{Effect of the grid size for bubble behavior}

The differences in the scales involved is one of the main challenges related to CFD modeling of multiphase systems. Mostly, the particles are in sub-millimeter range while the reactors are in scale of meters. Further, the computational grid can be in the scale of millimeters, centimeters or either in meters depending upon the size of the geometry and computational capacity. Unlike in packed beds, bubbling fluidized beds contain a dense particle phase and a dilute bubble phase. Therefore, the grid should be fine enough to capture the bubble properties as the bubbles play an important role in heat and mass transfer along with particle mixing inside the bed.

Grid $01,02 \ldots 05$ and a coarser grid having 2000 cells were simulated for 50 seconds in the bubbling fluidization regime. The time step of 0.001 seconds was used and a constant air velocity of $0,225 \mathrm{~m} / \mathrm{s}$ was maintained. The behavior of the bubbles in the $40^{\text {th }}$ second of the simulation are illustrated in Figure 13. Smaller and increased number of bubbles appears in the finer grids of grid 05 and 04 . The bubble size is becoming larger as the grid size is increased. Finally, the bubbles are almost disappeared at the coarsest grid with 2000 cells. Therefore, the grid should be fine enough to capture the localized bubble structures. In this case, grid 03 seems to be good enough because, the grid 04 and 05 produce almost the same bubble size.

\section{Conclusion}

The main objective of the paper was to analyze the effect of time step and grid size for the results in MP PIC modeling. The CPFD commercial package of Barracuda was used in this work. The results give a guidance about the critical parameters to be considered rather than presenting details with model validation.

The minimum fluidization velocity and pressure drop at minimum fluidization were greatly affected by the time step and however, it could be improved by increasing the simulation time. Time steps $0.005 \mathrm{~s}$ and $0.001 \mathrm{~s}$ produce the same of minimum fluidization velocity of $0.15 \mathrm{~m} / \mathrm{s}$ irrespective of the grid resolution. The bed pressure drop at bubbling fluidization regime was not affected considerably by the time step which is $12 \mathrm{pa} / \mathrm{mm}$. However, the minimum fluidization velocity could converge together when the simulation time for a particular air velocity was increased.

The grid size showed a minimal effect on the minimum fluidization velocity. However, the grid size had a great effect on the bubble size and consequently on the bed hydrodynamics. The close volume fraction was also found to be a deciding parameter in simulations for finding the minimum fluidization velocity.

Therefore, the simulation set up should be well optimized depending on the required accuracy of the results and availability of computer power. The physical parameters such as close volume fraction should be accurately measured and implemented in the simulations.

\section{Acknowledgements}

The authors like to forward their gratitude to University College of Southeast Norway for providing the Barracuda VR CFD package and computer resources for simulation. 


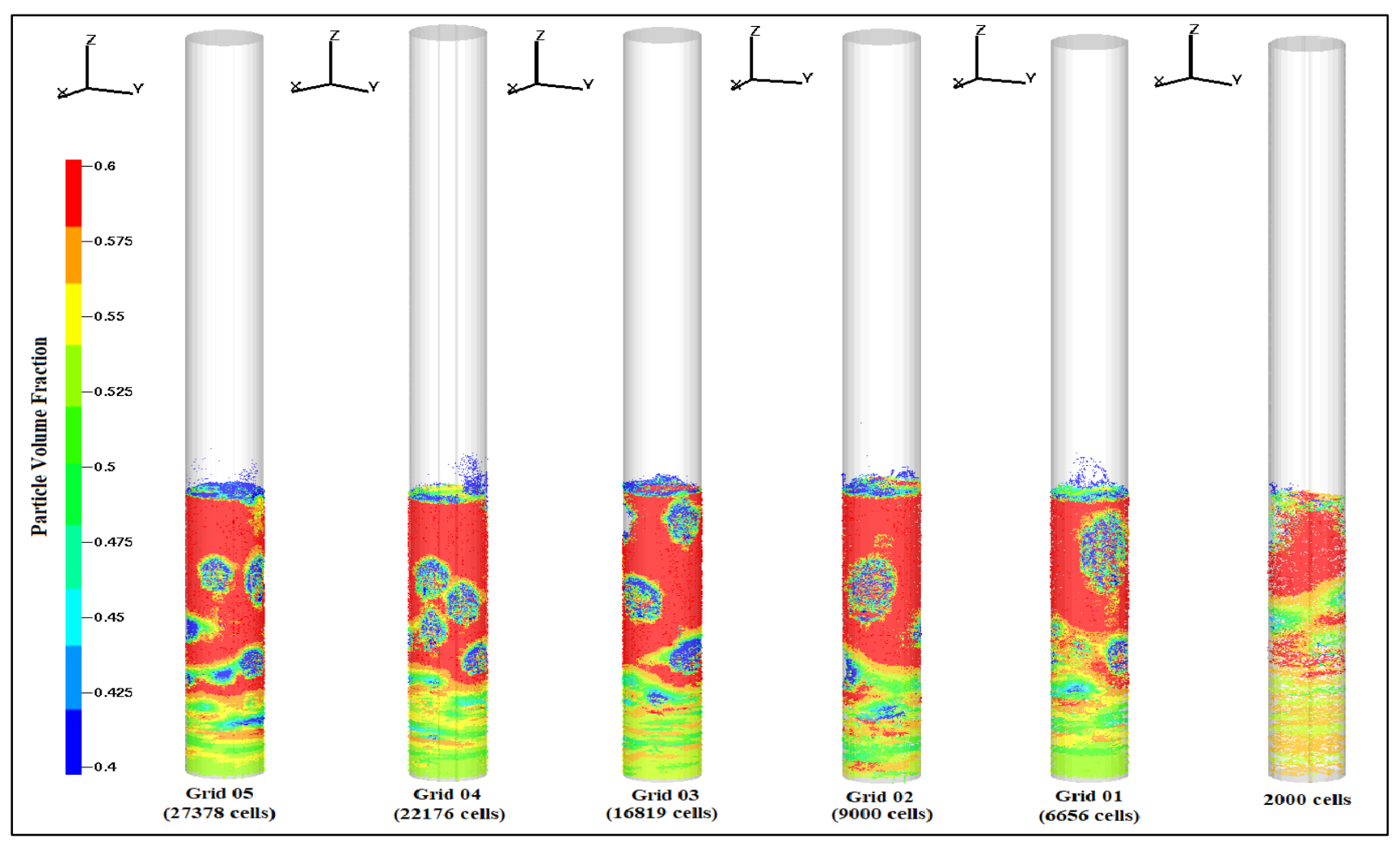

Figure 13. Effect of the computational grid size for the bubble size at $0.225 \mathrm{~m} / \mathrm{s}$ superficial air velocity

\section{References}

Andrews, M J, and P J O'Rourke. 1996. "The Multiphase Particle-in-Cell (MP-PIC) Method for Dense Particulate Flows." International Journal of Multiphase Flow 22 (2): 379-402.

Gidaspow. 1994, Multiphase Flow and Fluidization, Academic press

Esmaili, Ehsan, and Nader Mahinpey. 2011. "Adjustment of Drag Coefficient Correlations in Three Dimensional CFD Simulation of Gas-solid Bubbling Fluidized Bed." Advances in Engineering Software 42 (6): 375-86.

Geldart, D. 1973. "Types of Gas Fluidization." Powder Technology 7 (5): 285-92.

O'Rourke, Peter J, and Dale M Snider. 2012. "Inclusion of Collisional Return-to-Isotropy in the MP-PIC Method." Chemical Engineering Science 80: 39-54.

Snider, Dale M. 2007. "Three Fundamental Granular Flow Experiments and CPFD Predictions." Powder Technology 176 (1): 36-46.

Snider, D M. 2001. “An Incompressible Three-Dimensional Multiphase Particle-in-Cell Model for Dense Particle Flows." Journal of Computational Physics 170 (2): 52349.
Snider, D M, P J O'Rourke, and M J Andrews. 1998. "Sediment Flow in Inclined Vessels Calculated Using a Multiphase Particle-in-Cell Model for Dense Particle Flows." International Journal of Multiphase Flow 24 (8): 1359-82.

Souza Braun, Meire Pereira de, Andreza Tangerino Mineto, Hélio Aparecido Navarro, Luben Cabezas-Gómez, and Renato César da Silva. 2010. "The Effect of Numerical Diffusion and the Influence of Computational Grid over Gas-solid Two-Phase Flow in a Bubbling Fluidized Bed." Mathematical and Computer Modelling 52 (9): 13901402.

Vejahati, Farshid, Nader Mahinpey, Naoko Ellis, and Mehrdokht B. Nikoo. 2009. "CFD Simulation of GasSolid Bubbling Fluidized Bed: A New Method for Adjusting Drag Law." Canadian Journal of Chemical Engineering 87 (1): 19-30.

Bandara, J. C., Thapa, R. K., Moldestad, B. M. E., \& Eikeland, M. S. (2016). Simulation of Particle Segregation in Fluidized Beds Paper presented at the 9th EUROSIM Congress on Modelling and Simulation, Oulu, Finland. 